\title{
Acupuncture Research in the Era of Comparative Effectiveness Research
}

\author{
Remy R. Coeytaux, MD, PhD and \\ Duke Clinical Research Institute, Durham, North Carolina \\ Jongbae J. Park, DKM, PhD, LAc \\ Regional Center for Neurosensory Disorders and University of North Carolina at Chapel Hill, \\ Chapel Hill, North Carolina
}

\begin{abstract}
In this issue, Brinkhaus and colleagues (1) evaluate the effectiveness of acupuncture in the treatment of seasonal allergic rhinitis (SAR). They enrolled 422 individuals with SAR and IgE sensitization to birch and grass pollen in a multicenter trial in Germany. Patients were randomly assigned to receive 12 acupuncture treatments, 12 sham acupuncture treatments, or no acupuncture over 8 weeks between March and July. All patients were permitted to take the antihistamine medication cetirizine as a rescue medication, as well as an oral corticosteroid if symptoms were not adequately controlled with cetirizine. At the primary end point ( 8 weeks after randomization), the investigators found that acupuncture was associated with improvements of 0.7 point on the Rhinitis Quality of Life Questionnaire and 1.5 points in the rescue medication score compared with no acupuncture and improvements of 0.5 point on the Rhinitis Quality of Life Questionnaire and 1.1 points on the rescue medication score compared with sham acupuncture. A 1-point reduction in the rescue medication score is equivalent to a $10-\mathrm{mg} / \mathrm{d}$ reduction in cetirizine intake (2). This study builds on the investigators' earlier studies of acupuncture for SAR, including a pilot study that suggested that a combination of acupuncture and Chinese herbal medicine is superior to both sham acupuncture and placebo (3) and a pragmatic randomized, controlled trial that showed that a combination of acupuncture and Chinese herbal medicine is superior to sham acupuncture and placebo (4). Further evidence in support of acupuncture's effectiveness in treating allergic rhinitis is provided by a recent systematic review that concluded that acupuncture was cost-effective in Germany (5).
\end{abstract}

The purpose of the 3-group randomized, controlled trial design used by Brinkhaus and colleagues is 2-fold: to estimate the magnitude of effect (if any) associated with "true"

(C) 2013 American College of Physicians

Requests for Single Reprints: Remy R. Coeytaux, MD, PhD, Duke Evidence-based Practice Center, Duke Clinical Research Institute, PO Box 17969, Durham, NC 27715.

Current Author Addresses: Dr. Coeytaux: Duke Evidence-based Practice Center, Duke Clinical Research Institute, PO Box 17969, Durham, NC 27715.

Dr. Park: UNC Memorial Hospital, Department of Physical Medicine \& Rehabilitation, CB 7200, Room N1108, Chapel Hill, NC 27599-7200.

Disclaimer: The content is solely the responsibility of the authors and does not necessarily represent the official views of the National Institutes of Health.

Potential Conflicts of Interest: Disclosures can be viewed at www.acponline.org/authors/icmje/ConflictOfInterestForms.do? msNum=M13-0016. 
acupuncture relative to what might be expected in the absence of treatment (without consideration of placebo effects) and to determine whether a given course of acupuncture confers greater clinical benefit than a sham acupuncture protocol (for the purpose of controlling for placebo effects). The former is of interest to patients, clinicians, and payers who wish to be informed about expected outcomes associated with treatment versus no treatment, whereas the latter is intended to assess whether placebo effects may account for acupuncture's observed effects. In Brinkhaus and colleagues' study, acupuncture was shown to be more effective than both no acupuncture and sham acupuncture, but many other large clinical trials of acupuncture for other clinical conditions have shown it to be more effective than no acupuncture but not sham acupuncture. In those cases, the answer to the question of whether acupuncture is effective depends in large part on whether one considers a procedural-based intervention to be effective relative to the absence of that intervention, or relative to an artificial course of treatment that would never be administered in clinical practice and may not be physiologically inert.

To be considered effective, a pharmaceutical intervention generally needs to be proven more effective than placebo. Most surgical interventions, however, are not held to this standard. With some exceptions, including vertebral kyphoplasty (6) and knee arthroscopy (7), surgical interventions have not been subjected to testing by sham surgery-controlled trials. Acupuncture, on the other hand, has undergone extensive study with large, sham-controlled clinical trials. Over the past 15 years, there have been enough good-quality clinical trials of acupuncture to support patient-level meta-analyses for several clinical indications. One such recently published analysis of acupuncture for chronic pain conditions (back and neck pain, osteoar-thritis, headache, and shoulder pain) involving a total of 17922 patients showed that acupuncture was superior to both sham and no-acupuncture controls for each pain condition (8). In contrast, few, if any, surgical interventions have been shown to be more effective than a sham procedure in the context of a randomized, controlled trial.

Sham-controlled trials are an important tool in the evaluation of acupuncture's potential efficacy, but it may be time to prioritize studies that directly compare a specified acupuncture protocol with potentially therapeutic alternatives. There is precedence for evaluating the comparative effectiveness of a given intervention without first having demonstrated superiority over a sham intervention; for example, ample head-to-head studies have compared angioplasty with medical management in the treatment of coronary artery disease or cognitive behavior therapy with medical management for general anxiety. The argument to consider conducting fewer efficacy and more effectiveness trials of acupuncture may be especially timely, given current interest in patient-centered outcomes and comparative effectiveness research. For clinical conditions for which acupuncture has been shown to be compared with no acupuncture (but not necessarily compared with sham acupuncture), it may be time to begin asking such questions as: How does acupuncture compare directly with other therapeutic approaches? Which of the many acupuncture traditions or approaches is most effective or appropriate for a given clinical indication? What outcomes or process measures should we be assessing in clinical trials of acupuncture? Is the magnitude of effect, if any, associated with acupuncture for a given clinical indication "worth it" from the perspective of patients, payers, or policymakers? 
The study by Brinkhaus and colleagues represents a step in this direction, in part because the investigators used an acupuncture protocol that simulates actual clinical practice. This is important because many investigators in the past have prioritized internal validity over external validity by designing the true acupuncture protocol to resemble the sham acupuncture control, thereby possibly underestimating the effect of acupuncture as practiced in real-world settings. With this study lending compelling support to the effectiveness of real-world acupuncture for SAR, we envision further research that uses patient-centered outcomes and comparative effectiveness research designs to inform patients, clinicians, policymakers, payers, and other stakeholders of the role that acupuncture might play in our health care system.

\section{Acknowledgments}

Financial Support: Dr. Park was supported by the National Institute of Dental and Craniofacial Research of the National Institutes of Health (award K12DE022793).

\section{References}

1. Brinkhaus B, Ortiz M, Witt CM, Roll S, Linde K, Pfab F, et al. Acupuncture in patients with seasonal allergic rhinitis. A randomized trial. Ann Intern Med. 2013; 158:225-34. [PubMed: 23420231]

2. Kuehr J, Brauburger J, Zielen S, Schauer U, Kamin W, Von Berg A, et al. Efficacy of combination treatment with anti-IgE plus specific immunotherapy in polysensitized children and adolescents with seasonal allergic rhinitis. J Allergy Clin Immunol. 2002; 109:274-80. [PubMed: 11842297]

3. Brinkhaus B, Hummelsberger J, Kohnen R, Seufert J, Hempen CH, Leonhardy H, et al. Acupuncture and Chinese herbal medicine in the treatment of patients with seasonal allergic rhinitis: a randomized-controlled clinical trial. Allergy. 2004; 59:953-60. [PubMed: 15291903]

4. Brinkhaus B, Witt CM, Jena S, Liecker B, Wegscheider K, Willich SN. Acupuncture in patients with allergic rhinitis: a pragmatic randomized trial. Ann Allergy Asthma Immunol. 2008; 101:53543. [PubMed: 19055209]

5. Kallmes DF, Comstock BA, Heagerty PJ, Turner JA, Wilson DJ, Diamond TH, et al. A randomized trial of vertebroplasty for osteoporotic spinal fractures. N Engl J Med. 2009; 361:569-79. [PubMed: 19657122]

6. Moseley JB, O'Malley K, Petersen NJ, Menke TJ, Brody BA, Kuykendall DH, et al. A controlled trial of arthroscopic surgery for osteoarthritis of the knee. N Engl J Med. 2002; 347:81-8. [PubMed: 12110735]

7. Kim SY, Lee H, Chae Y, Park HJ, Lee H. A systematic review of cost-effectiveness analyses alongside randomised controlled trials of acupuncture. Acupunct Med. 2012; 30:273-85. [PubMed: 23099289]

8. Vickers AJ, Cronin AM, Maschino AC, Lewith G, MacPherson H, Foster NE, et al. Acupuncture Trialists' Collaboration. Acupuncture for chronic pain: individual patient data meta-analysis. Arch Intern Med. 2012; 172:1444-53. [PubMed: 22965186] 